\title{
Surface segregation of bimetallic alloys in nanoscale confinement
}

\author{
Ming Hu, ${ }^{1,3, a)}$ Konstantinos P. Giapis, ${ }^{2, b)}$ Javier V. Goicochea, ${ }^{3}$ and Dimos Poulikakos ${ }^{1, c)}$ \\ ${ }^{1}$ Department of Mechanical and Process Engineering, Laboratory of Thermodynamics in Emerging \\ Technologies, ETH Zurich, 8092 Zurich, Switzerland \\ ${ }^{2}$ Division of Chemistry and Chemical Engineering, California Institute of Technology, Pasadena, \\ California 91125, USA \\ ${ }^{3}$ Zurich Research Laboratory, IBM Research GmbH, 8803 Rüschlikon, Switzerland
}

(Received 21 July 2010; accepted 15 September 2010; published online 12 October 2010)

\begin{abstract}
The surface segregation of Pt atoms in liquid bimetallic alloys confined in carbon nanotube cavities was studied using molecular dynamics simulations. Considerable enrichment in the Pt-atom surface density was found to occur in Pt alloys, when the complementary metal has surface energy higher than Pt and simultaneously metal-wall interaction strength lower than that of Pt with the confining wall. The results suggest that solidification of liquid binary alloys in nanochannels could produce core-shell nanorods with the shell enriched in one of the components for catalytic and other applications. (C) 2010 American Institute of Physics. [doi:10.1063/1.3500825]
\end{abstract}

The influence of nanoscale confinement on the thermodynamic behavior and physical properties of materials has attracted widespread interest in the last two decades. ${ }^{1}$ Constraints in the packing of atoms or molecules confined in one dimension could influence the freezing of liquids and lead to the formation of new solid phases. ${ }^{2}$ Carbon nanotubes (CNTs), with their hollow inner cavity, offer an ideal nanochannel for performing such studies. ${ }^{2,3}$ Indeed, both helical strand and cylindrical shell structures-not present in bulk phases-have been demonstrated for single-element materials confined in CNTs. ${ }^{4-6}$ Pure metals, in particular, were predicted to form cylindrically layered structures. ${ }^{7,8} \mathrm{Al}-$ though they have received markedly less attention, metallic alloys should also form cylindrical shell structures when solidified in CNTs. However, differences in the interaction energy between the alloy components and the graphitic walls may allow one element to accumulate near the wall while the other is displaced toward the tube center. This surface segregation process may lead to the spontaneous formation of core-shell nanostructures from impure mixtures, with possibly unexpected electrical, magnetic, or catalytic properties.

Here we report on the propensity of the Pt atoms to segregate near confining walls in Pt-based metallic alloys. We simulate the solidification of binary liquid mixtures inside CNTs using molecular dynamics. We seek mixtures of low initial Pt concentration for which the surface density of the segregated $\mathrm{Pt}$ atoms is maximized. Our model structure consists of a binary alloy column confined in a $(20,20)$ single-walled CNT, which is composed of 7680 carbon atoms (diameter $=2.7 \mathrm{~nm}$, length $=23.4 \mathrm{~nm}$ ). We consider various Pt-based alloys and focus on the effect of the initial concentration, metal-wall interaction, and surface energy on the quality of the final core-shell structure. The alloy column (initially $\sim 18$-nm-long) is taken from the equilibrium configuration for bulk alloy of the same composition. The alloy column has two free ends and contains $\sim 4000$ atoms with $\mathrm{Pt}$ concentration ranging from 5 to 95 at. \%. The entire system

\footnotetext{
a) Author to whom correspondence should be addressed. Electronic mail: hum@ethz.ch.

b)Electronic mail: giapis@cheme.caltech.edu.

${ }^{c)}$ Electronic mail: dimos.poulikakos@sl.ethz.ch.
}

is confined within a rectangular simulation box of $8 \times 23.4$ $\times 8 \mathrm{~nm}^{3}$, long enough in the $x$ and $z$ directions to avoid self-imaging interactions. Periodic boundary conditions are used in all directions, resulting in an alloy nanorod of finite length confined in an infinitely long CNT with the tube axis extending along the $y$ direction.

Among the potentials ${ }^{9-11}$ used in the literature to simulate fcc metals such as $\mathrm{Al}, \mathrm{Ni}$, and $\mathrm{Pt}$, we chose the quantum Sutton-Chen (QSC) potential. ${ }^{11}$ The QSC includes quantum corrections and takes into account the zero point energy, allowing for better prediction of temperature-dependent properties and, in particular, of the phase transformation of fcc alloys. ${ }^{12-14}$ The relatively weak carbon-metal interaction is modeled by pairwise Lennard-Jones (LJ) potentials, with energy and distance parameters obtained from the geometric and arithmetic mean, respectively, of the corresponding LJ parameters for graphite ${ }^{15}$ and the considered metal. ${ }^{16}$ Metalmetal interactions and van der Waals forces were both truncated at $10 \AA$. A static CNT cavity with fixed C-atom positions was used to reduce the computational load. The LAMMPS (Ref. 17) package was used with a time step of $1 \mathrm{fs}$ throughout. Initially, the system was equilibrated in an ensemble with a fixed number of atoms $N$ and volume $V$. A Nosé-Hoover temperature thermostat of $T=2900 \mathrm{~K}$ was applied for $10 \mathrm{~ns}$ with a relaxation time of $0.1 \mathrm{ps}$ to ensure full mixing in the molten column. The system was further relaxed for 2 ns with NVE ensemble (constant volume, no thermostat). Subsequently, the system was cooled down in steps of $100 \mathrm{~K}$ at a rate of $25 \mathrm{~K} / \mathrm{ns}$, with an additional relaxation of $4 \mathrm{~ns}$ at each step. ${ }^{18}$ The liquid alloy solidified between 1800-1000 K, depending on the initial Pt composition. All structures were cooled down to $500 \mathrm{~K}$, where they were analyzed.

Snapshots of crystallized $\mathrm{Pt}-\mathrm{Ni}$ alloy nanorods are shown in Fig. 1 for four alloy compositions. A core-shell structure forms spontaneously in all cases, with a Ni-rich core and a Pt-rich shell. The surface pattern varies considerably as the Pt content increases. The segregation of Pt atoms to the surface is apparent even at low Pt concentrations ( $\leq 15$ at. \%), though in this case the majority of surface atoms are $\mathrm{Ni}$ [Fig. 1(a)]. At higher $\mathrm{Pt}$ concentrations ( $\geq 25$ at. \%), the segregation of Pt atoms to the confining 
(a)

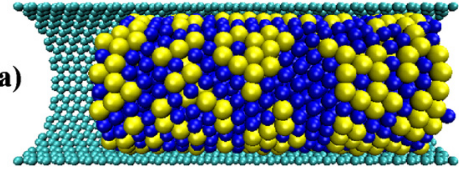

(b)

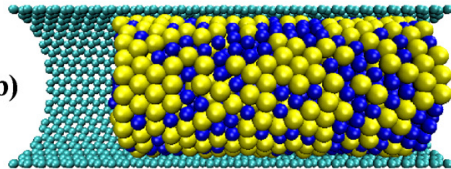

(c)

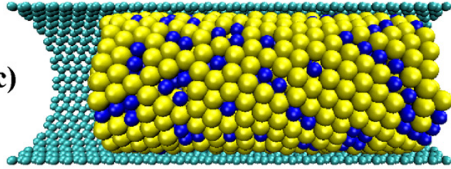

(d)

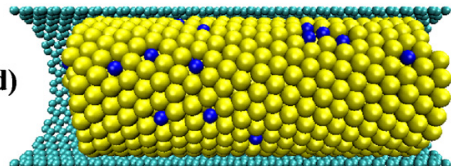

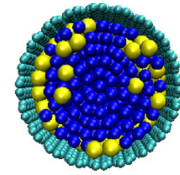
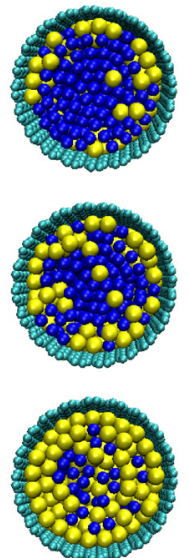

FIG. 1. (Color online) Snapshots of crystallized Pt-Ni nanorods at $500 \mathrm{~K}$ in side view along the nanotube center axis. Only half of the system is shown. (Right) Cross-sectional views at the nanorod middle. From (a) to (d): initial concentration of Pt 15 at. \%, 25 at. \%, 40 at. \%, and 75 at. \%, respectively. Color coding: dark: Ni; light: Pt; and medium: $\mathrm{C}$.

wall becomes more pronounced. For example, the nanorod formed from the initial 40 at. \% Pt liquid alloy has an outermost layer that consists of 84 at. \% Pt [Fig. 1(c)].

While atom ordering near the walls is responsible for the formation of the core-shell structure, surface segregation allows control of the composition of the shell at the monolayer level. This spontaneous process could offer a tractable way to synthesize core-shell nanorods from impure alloys with the surface layer enriched in a particular metal. Such structures could be useful in catalysis offering better utilization of the catalytic element. Notably, the cylindrical organization of the atoms on a surface of large curvature could lead to modified catalytic properties. While segregation occurs spontaneously, the proposed method requires that a liquid alloy be introduced into nanochannels, a difficulty that may be overcome by wetting or electrowetting from the melt, or by using nanoparticles with subsequent melting. Once the structure is solidified, the template would be removed chemically. CNTs may be removed by exposure to atomic oxygen. ${ }^{19}$

The radial density profiles, shown in Fig. 2, provide further insight into the core-shell structures formed. For comparison, the atom density of pure $\mathrm{Pt}$ in a $(20,20) \mathrm{CNT}$ is also shown [Fig. 2(d)]. Average densities are calculated for 8-nmlong cylindrical segments from the middle of the nanorod. As expected, a cylindrically layered structure is found, similar to that observed in pure metal nanowires. ${ }^{5,8}$ Both Pt and Ni peaks have almost the same position due to the similarity of their corresponding QSC potentials. ${ }^{20}$ For all compositions shown, a Pt-rich layer is formed on the nanorod outer surface. At higher Pt concentrations, more Pt atoms segregate thus enriching not only the outermost layer but also adjacent layers [see Figs. 2(b)-2(d)]. For the alloy with 75 at. \% Pt, the nanorod surface consists of almost pure Pt.

Surface segregation depends not only on the alloy composition, confinement size, and metal-wall interaction strength, but also on surface energy, mixing energy, and mixing entropy of the alloy components. The influence of these

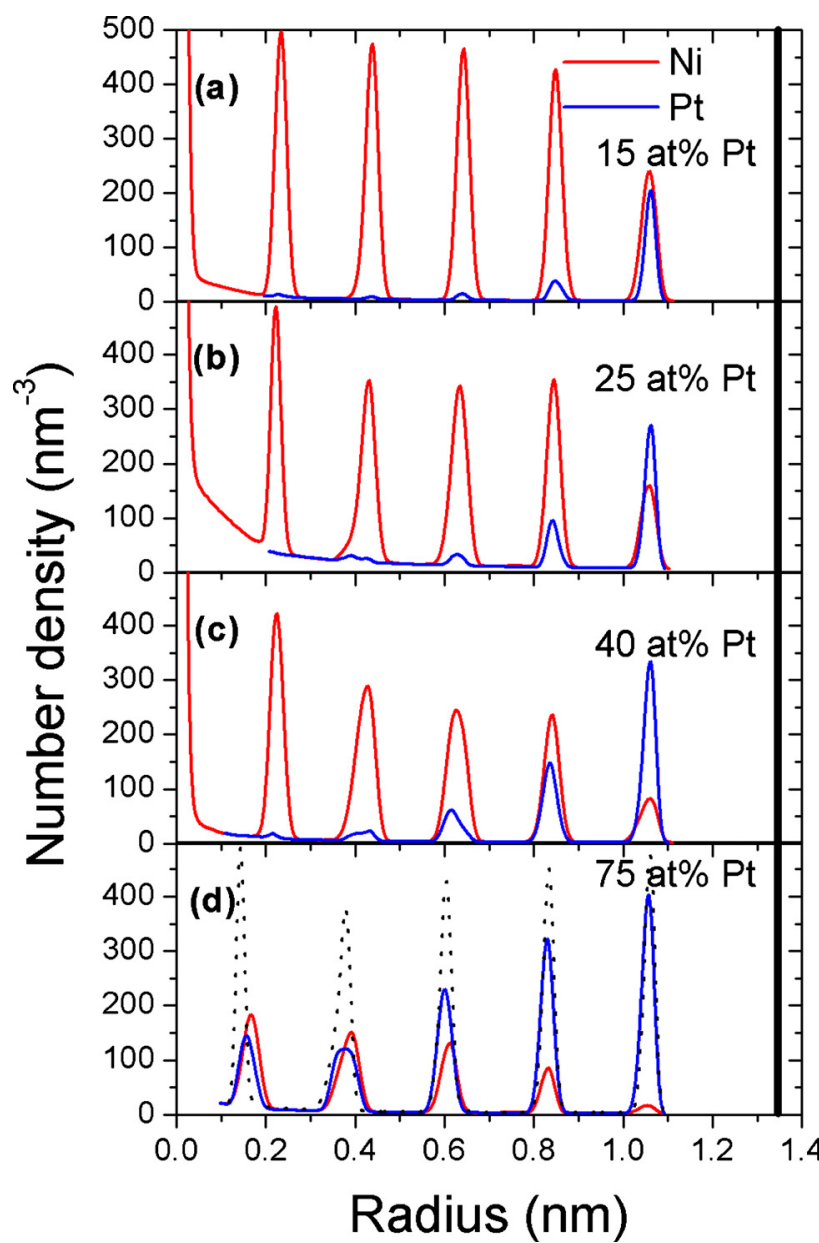

FIG. 2. (Color online) Equilibrated radial density profiles of crystallized Pt-Ni structures in $(20,20)$ CNT. From (a) to (d): initial concentration of Pt 15 at. $\%, 25$ at. $\%, 40$ at. $\%$, and 75 at. \%, respectively. The atom density of pure Pt crystallized in the same CNT is also shown (dotted line in last panel). The black vertical line at a radius of $1.35 \mathrm{~nm}$ indicates the position of the CNT wall.

parameters on segregation was assessed by considering mixtures of $\mathrm{Pt}$ with $\mathrm{Ir}, \mathrm{Ni}$, and $\mathrm{Al}$, which have surface energies higher than, comparable to, and lower than Pt, respectively, as predicted by the Sutton-Chen model. ${ }^{20}$ Results for these

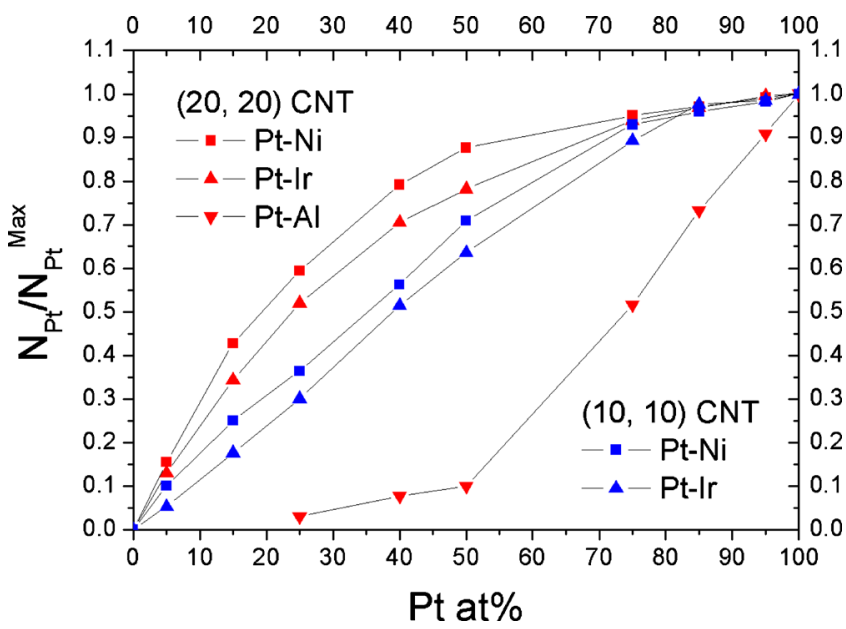

FIG. 3. (Color online) Normalized surface density $\left(N_{\mathrm{Pt}} / N_{\mathrm{Pt}}^{\mathrm{Max}}\right)$ of segregated $\mathrm{Pt}$ atoms as a function of the initial $\mathrm{Pt}$ concentration in solidified alloy nanorods. Color coding: light: alloy in $(10,10)$ CNT; dark: alloy in $(20,20)$ CNT. Chart symbols: $\square$ Pt-Ni; $\boldsymbol{\Delta}$ Pt-Ir; and $\boldsymbol{\nabla}$ Pt-Al. 


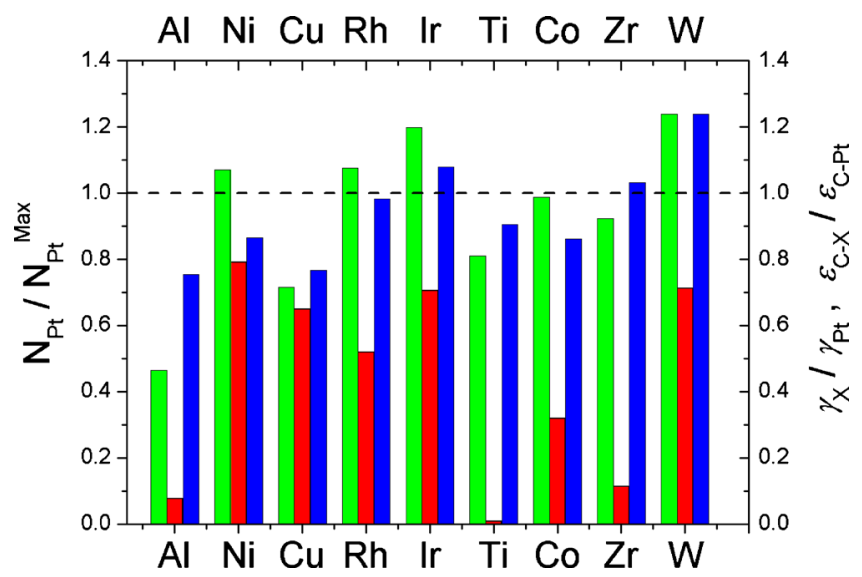

FIG. 4. (Color online) Comparison of surface density of segregated Pt atoms for selected Pt-X alloys with initial Pt concentration of 40 at. \%. $\mathrm{X}$ is the metal indicated on the abscissa. Color coding: left bar: relative surface energy between two elements; middle bar: normalized surface density of the segregated Pt; and right bar: relative metal-C interaction strength.

alloys are shown in Fig. 3 in terms of the normalized ${ }^{21} \mathrm{Pt}$ surface atom density on a solidified alloy nanorod. It is clearly seen that the maximum Pt segregation occurs for the $\mathrm{Pt}-\mathrm{Ni}$ alloy. To explain this result, let us denote the secondary element by X. Pt segregation out of the Pt-X alloy depends not only on the relative value of the component surface energy (denoted as $\gamma_{\mathrm{Pt}}$ and $\gamma_{\mathrm{X}}$ ) but also on the relative metal-C interaction strength $\left(\varepsilon_{\mathrm{C}-\mathrm{Pt}}\right.$ and $\left.\varepsilon_{\mathrm{C}-\mathrm{X}}\right)$. Generally, the larger the surface energy of $\mathrm{X}$, the easier it is for $\mathrm{Pt}$ to be segregated out. On the other hand, the larger the $\mathrm{X}-\mathrm{C}$ interaction strength, the more difficult it is for $\mathrm{Pt}$ to displace $\mathrm{X}$ atoms from the surface. Since the metal-C interaction strength is usually proportional to the surface energy of the metal, a compromise between the surface energy of $\mathrm{X}$ and $\mathrm{X}-\mathrm{C}$ interaction strength appears necessary to optimize Pt segregation. As mentioned before, $\gamma_{\mathrm{Al}}<\gamma_{\mathrm{Pt}}<\gamma_{\mathrm{Ni}}<\gamma_{\mathrm{Ir}}$ but $\varepsilon_{\mathrm{C}-\mathrm{Al}}<\varepsilon_{\mathrm{C}-\mathrm{Ni}}<\varepsilon_{\mathrm{C}-\mathrm{Pt}}<\varepsilon_{\mathrm{C}-\mathrm{Ir}}$ Of the three alloys, the Pt-Ni exhibits the best performance because it has both larger $\gamma_{\mathrm{X}}$ and lower $\varepsilon_{\mathrm{C}-\mathrm{X}}$ than the corresponding parameters for Pt. Note that $\mathrm{Al}$, with the smallest surface energy and metal-C interaction strength, produces the opposite behavior in the $\mathrm{Pt}-\mathrm{Al}$ alloy. Increased confinement was also considered for the $\mathrm{Pt}-\mathrm{Ir}$ and $\mathrm{Pt}-\mathrm{Ni}$ alloys by simulating solidification in a $(10,10)$ CNT. Both alloys show (Fig. 3) a dramatic reduction in Pt atom segregation as compared to the $(20,20) \mathrm{CNT}$, as a consequence of the more severe confinement.

Other candidates ${ }^{22}$ were also considered as alloy components for enhanced Pt segregation. The results for a fixed Pt alloy composition of 40 at. $\%$ in a $(20,20)$ CNT are com- pared as bar charts in Fig. 4, where the metal surface energy and metal-C interaction strength are also shown. Again, the $\mathrm{Pt}-\mathrm{Ni}$ alloy offers the highest surface density of segregated Pt. Note that $\mathrm{Ni}$ is the only metal, which satisfies simultaneously the following two conditions: $\gamma_{\mathrm{X}}>\gamma_{\mathrm{Pt}}$ and $\varepsilon_{\mathrm{C}-\mathrm{X}}$ $<\varepsilon_{\mathrm{C}-\mathrm{Pt}}$. Being naturally abundant and cheap, Ni should make an ideal alloy component to $\mathrm{Pt}$ for segregation experiments, if a method for introducing and solidifying Pt-Ni alloys in CNTs could be realized.

K.P.G. is grateful to ETH-Zürich for a visiting professorship grant. Computational support from the Brutus Cluster at ETH-Zurich is acknowledged. This work was supported by the Swiss National Supercomputing Centre-CSCS under project ID s243.

${ }^{1}$ C. Alba-Simionesco, B. Coasne, G. Dosseh, G. Dudziak, K. E. Gubbins, R. Radhakrishnan, and M. Sliwinska-Bartkowiak, J. Phys.: Condens. Matter 18, R15 (2006).

${ }^{2}$ P. Ball, Nature (London) 361, 297 (1993).

${ }^{3}$ D. Ugarte, A. Chatelain, and W. A. de Heer, Science 274, 1897 (1996).

${ }^{4}$ X. Fan, E. C. Dickey, P. C. Eklund, K. A. Williams, L. Grigorian, R. Buczko, S. T. Pantelides, and S. J. Pennycook, Phys. Rev. Lett. 84, 4621 (2000)

${ }^{5}$ S. Arcidiacono, J. H. Walther, D. Poulikakos, D. Passerone, and P. Koumoutsakos, Phys. Rev. Lett. 94, 105502 (2005).

${ }^{6}$ L. H. Guan, K. Suenaga, Z. Shi, Z. Gu, and S. Iijima, Nano Lett. 7, 1532 (2007).

${ }^{7}$ W. Y. Choi, J. W. Kang, and H. J. Hwang, Phys. Rev. B 68, 193405 (2003).

${ }^{8}$ A. Kutana and K. P. Giapis, Phys. Rev. B 76, 195444 (2007).

${ }^{9}$ S. M. Foiles, M. I. Baskes, and M. S. Daw, Phys. Rev. B 33, 7983 (1986).

${ }^{10}$ A. P. Sutton and J. Chen, Philos. Mag. Lett. 61, 139 (1990).

${ }^{11}$ T. Cagin, Y. Kimura, Y. Qi, L. Yue, I. Hao, H. Ikeda, W. L. Johnson, and W. A. Goddard, Bulk Metallic Glasses, MRS Symposia Proceedings No. 554 (Materials Research Society, Pittsburgh, 1999), p. 43.

${ }^{12}$ X. Liu, C. G. Meng, and C. H. Liu, Phase Transitions 79, 249 (2006).

${ }^{13}$ Z. A. Tian, R. S. Liu, C. X. Zheng, H. R. Liu, Z. Y. Hou, and P. Peng, J. Phys. Chem. A 112, 12326 (2008).

${ }^{14}$ Y. H. Wen, Y. Zhang, J. C. Zheng, Z. Z. Zhu, and S. G. Sun, J. Phys. Chem. C 113, 20611 (2009).

${ }^{15}$ V. R. Bhethanabotla and W. A. Steele, Phys. Rev. B 41, 9480 (1990).

${ }^{16}$ S. Zhen and G. J. Davies, Phys. Status Solidi A 78, 595 (1983).

${ }^{17}$ S. Plimpton, J. Comput. Phys. 117, 1 (1995).

${ }^{18}$ For some cases, the relaxation time was doubled to ensure that the system had reached equilibrium.

${ }^{19}$ A. Kutana and K. P. Giapis, J. Phys. Chem. C 113, 14721 (2009).

${ }^{20}$ B. D. Todd and R. M. Lynden-Bell, Surf. Sci. 281, 191 (1993).

${ }^{21}$ The surface density $N_{\mathrm{Pt}}^{\mathrm{Max}}$ of a nanorod formed from pure Pt in the same size CNT is used as the normalization constant. The $N_{\mathrm{Pt}}^{\mathrm{Max}}$ values for pure Pt in $(10,10)$ and $(20,20)$ CNTs have been calculated to be $8.53 \mathrm{~nm}^{-2}$ and $11.94 \mathrm{~nm}^{-2}$, respectively.

${ }^{22}$ For alloys consisting hep and bcc metals, we adopted the potential proposed by X. W. Zhou, H. N. G. Wadley, R. A. Johnson, D. J. Larson, N. Tabat, A. Cerezo, A. K. Petford-Long, G. D. W. Smith, P. H. Clifton, R. L. Martens, and T. F. Kelly, Acta Mater. 49, 4005 (2001). 